\title{
Editorial: The Newly Launched International Journal of Analytical Mass Spectrometry and Chromatography
}

\author{
Ilia Brondz ${ }^{1,2}$ \\ ${ }^{1}$ Department of Biosciences, University of Oslo, Oslo, Norway \\ ${ }^{2}$ R\&D Department of Jupiter Ltd., Ski, Norway \\ Email: ilia.brondz@bio.uio.no, ilia.brondz@gmail.com
}

Received July 1, 2013; revised August 4, 2013; accepted September 1, 2013

\begin{abstract}
Copyright (C) 2013 Ilia Brondz. This is an open access article distributed under the Creative Commons Attribution License, which permits unrestricted use, distribution, and reproduction in any medium, provided the original work is properly cited.
\end{abstract}

We are now launching the first issue of the first volume of the International Journal of Analytical Mass Spectrometry and Chromatography (IJAMSC). This newly launched journal is an open access journal, whose primary aim is to provide a platform for researchers and practitioners, worldwide, to promote, share, and discuss various new issues and developments in all areas of Mass Spectrometry and Chromatography, as well as theoretical developments in all areas related to this dynamic and developing field of the sciences. We hope to become an example to other open access journals in Scientific Research Publishing, Inc., and to journals beyond the boundaries of our parent corporation. We aim to set an example as a progressive organization that helps authors to share their interests, results, and opinions with other researchers and with readers from the general public, industry, and government authorities. We also intend to cooperate with universities, research institutes, industry, and government authorities to promote progressive ideas and information, and to curb plagiarism, falsifications, and disinformation, such as the cases disclosed in [1].

We will resurrect important institutions of journalistic practice, such as a "Letters to the Editor" the section, which has been done away with by many publishers, such as those that follow the example of Elsevier; in the attempts to conceal mistakes and the disability of editors. The reason for the decision by one Elsevier journal to abolish its "Letters to the Editor" section is described in [1] on p. 184 as follows: "The chief editor of Journal of Pharmaceutical and Biomedical Analysis (JPBA) Mr. Bezhan Chankvetadze when confronted with the "Letters to the Editor" regarding cases of plagiarism and inappropriate performance in review and editorial work, went so far as to abolish the "Letter to the Editor" section in the journal, thus removing this forum...".

The suggestion of direct corruption, in terms of "The close relationship the publisher, Elsevier, has with pharmaceutical companies", is highlighted in the article "El- sevier published 6 fake journals”, which is posted online by Bob Grant: http://www.the-scientist.com/blog /display/55679/\#ixzz0mmsPoMlS. In the article, the writer states: "Scientific publishing giant Elsevier put out a total of six publications between 2000 and 2005 that were sponsored by unnamed pharmaceutical companies and looked like peer reviewed medical journals, but did not disclose sponsorship, the company has admitted...”. Elsevier did not deny colluding with the pharmaceutical industry. For more detail about cases of inappropriate editorials and publications, see [1].

The above examples show that readers are not protected from disinformation by well-established publishers or high impact factor journals.

We will do our best to present in our publications the truth, the whole truth, and nothing but the truth. It is our slogan. However, we will not prevent discussion or the expression of new ideas, facts, and contradictions, as only through discussion and argument can scientific facts be born and tested.

IJAMSC welcomes papers relevant to the development and practice of sample preparation and specific derivatization for mass spectrometry (MS) and chromatography, and the connection of mass spectrometers to other analytical instrumentation for providing high accuracy measurements, such as in Gas Chromatography-Mass Spectrometry (GC-MS), Gas Chromatography-Mass Spectrometry with Supersonic Molecular Beams (GC-MS with SMB), High-Speed Gas Chromatography-Mass Spectrometry (HSGC-MS), Supercritical Fluid ChromatographyMass Spectrometry (SFC-MS), and Liquid Chromatography-Mass Spectrometry (LC-MS). We also welcome research using such instrumentation for molecular structure elucidation in organic and inorganic molecules. The use of MS equipment as a universal detector in multidetection systems for enhanced recording and elucidation of the detailed composition of natural substances is demanding; hence, both natural and synthetic samples will be of interest. This technique is described in the paper 
"Supercritical fluid chromatography of secondary metabolites and multi-analysis by mass spectrometry-ultraviolet and corona charged aerosol detection” $[2,3]$.

\section{Mass Spectrometry}

Mass spectrometry is widely discussed in the literature as a detection tool and in relation to analytical methods of separation, such as GC, SCF, high performance liquid chromatography (HPLC), capillary electrophoresis (CE), thin layer chromatography (TLC) and as an analytical tool for the determination of molecular structures. MS is a powerful tool for structural analysis. Alone, MS is not enantiospecific, but in combination with HPLC, SFC, GC, CE, or even TLC, it can adequately demonstrate the presence of isomers and enantiomers. The recording by MS of enantiomers in a mixture is possible only by using chromatography with chiral columns or if chiral selectors are used in the resolution process. MS is an excellent tool for the evaluation of the constitutional, cis- or trans-, geometric, or other isomers, alone or in conjunction with chromatography. By using fastseparation techniques in conjunction with MS, it is possible to monitor syntheses or the dynamics of reactions.

Improvements in the construction of MS apparatuses are of significant importance, together with the development and improvement of software and the analytic methodology.

From its original primitive form, MS instrumentation has been developed, step by step, into the super-modern, complex, multitasking equipment of today. The first mass spectrometers were of the single-focus type, in which the positive ions were deflected through $180^{\circ}$ sector in a magnetic field, $\mathrm{H}$. The focused ion beams were recorded on a photographic plate.

The first mass spectrometer was developed by Arthur J. Dempster and Francis W. Aston in 1918/19 [4]. Their invention was actually a mass spectrograph. Arthur Dempster was born in Canada in 1886, and studied in Canada, Germany, and the USA. He conducted his research at the University of Chicago from 1916 until his death in 1950. Besides the development of MS, he is known for the discovery of the uranium isotope ${ }^{235} \mathrm{U}$. He was also a member of the Manhattan Project. Francis Aston was born in Birmingham in 1877, and in 1893 enrolled at the University of Birmingham, where he studied physics under John Henry Poynting and chemistry under Frankland and Tilden.

However, the history of MS actually begins with a discovery made by a German scientist, Eugen Goldstein (1850-1930). In 1886, he observed rays in gas discharges under low pressure. He discovered that tubes with a perforated cathode emitted a glow at the cathode end. The rays propagated from the anode and through the channels in the perforated cathode, in a direction opposite to that taken by charged cathode rays. He called these rays “Kanalstrahlen” or “canal rays”. In 1899, the German physicist Wilhelm Wien (Wilhelm Carl Werner Otto Fritz Franz Wien, 1864-1928) found that electric or magnetic fields could deflect the canal rays, and he constructed a device that separated the positive rays according to their charge-to-mass ratio. The rays were positively charged particles-later found to be ions. The behavior of ions in a homogeneous, linear, static magnetic field obeys the Lorentz force law, and this holds true in a sector instrument. The Lorentz equation, given below, is fundamental to all MS techniques:

$$
F=q\left(E+v^{\prime} B\right),
$$

where $E$ is the strength of the electric field, $B$ is the induction of the magnetic field, $q$ is the particle's electric charge, and $v$ is the particle's velocity.

An ion with a charge $e$ accelerated through a voltage $V$ will acquire a translational (kinetic) energy equal to $\mathrm{eV}$. Therefore, the kinetic energy of an ion is independent of its mass. Because $1 / 2 m v^{2}=e V$, where $m$ is the mass of the ion and $v$ is the magnitude of its velocity after its acceleration in an electric field; more massive ions will travel more slowly. Ions will be accelerated under the influence of a magnetic field of magnitude $B$. The magnitude of the acceleration is $v^{2} / r$, and it is directed perpendicular to the particle's movement, which implies that the particle will describe a circular trajectory with radius $r$. From Newton's second law of motion and the Lorentz force law, it follows that

$$
\mathrm{BeV}=m v^{2} / r \text {. }
$$

Combining this equation with $1 / 2 m v^{2}=e V$ results in

$$
m / e=B^{2} r^{2} / 2 V .
$$

From this equation, it follows that for a given magnetic field strength and accelerating voltage, ions with a given $m / e$ ratio will follow a distinct path of radius $r$, where $r$ is determined by $m / e$. It is possible to sweep the ions of various $m / e$ ratios past the exit slit, either by varying $B$ while holding $V$ constant, or by varying $V$ while holding $B$ constant. The first technique is called "magnetic scanning”, and the second technique is called "electric scanning" or "voltage scanning".

It is not necessary to have an angle of deflection of $180^{\circ}$. A $90^{\circ}$ sector field instrument is less expensive and easier to produce. It also has the advantage that the ion source and collector are more accessible than in the $180^{\circ}$ sector version of the instrument.

A $90^{\circ}$ sector field instrument should be seen as a modern version of the original instrument developed by Dempster. The general problem with these instruments is that the resolving power is limited by the initial spread of the translational energies of the ions on leaving the source. 
The initial spread of the translational energies depends on the Boltzmann distribution and the nonhomogeneous field in the source. These problems were overcome in the apparatus constructed by Mattauch and Herzog [5] by passing the ions through an electric field before their passage through the magnetic field. In Mattauch and Herzog's instrument, the electrostatic analyzer had a sector of $31^{\circ} 82^{\prime}$ and the magnetic analyzer had a sector of $90^{\circ}$. The $r_{1}$ was the minimum focal radius and the $r_{2}$ was the maximum focal radius. In this instrument, photographic plates were used to record the ions. The photographic plates were placed at $45^{\circ}$ relative to the $r_{2}$. This method of analysis is called "mass spectroscopy".

In modern instruments, electronic devices are used for precise and sensitive detection; these instruments are called "mass spectrometers" and the method is "mass spectrometry". Later, in 1953, Johnson and Nier published an article describing another configuration of sectors in a MS instrument [6]. Accounts that give more detail on the sectors in MS instruments are available in the literature $[7,8]$. In the instrument of Johnson and Nier, the electrostatic analyzer field is $90^{\circ}$ and the magnetic analyzer field is $60^{\circ}$. There are several other types of MS instruments, such as the Hinterberger-Konig mass spectrometer, with a $42^{\circ} 3^{\prime}$ electric sector and a $130^{\circ}$ magnetic sector. Mass spectrometers can have also a Takeshita geometry, with a $54^{\circ} 43^{\prime}$ electric sector and a $180^{\circ}$ magnetic sector, a Matsuda geometry, with an $85^{\circ}$ electric sector and a $72^{\circ} 50$ ' magnetic sector; or a Bainbridge-Jordan geometry, with a $127^{\circ} 30^{\prime}$ electric sector and a $60^{\circ}$ magnetic sector, with a resolving power of 600 and a relative precision of one part in $10,000[9,10]$.

The resolving power and relative precision of a specific instrument are very important characteristics when recording ions with low m/e ratios. For verification of the isomeric differences between primaquine and its contaminating agent, quinocide, an instrument with high precision and high resolving power was used to record the $m / e 44$ ions [11].

The ability of a MS instrument to produce high quality, informative spectra also depends on the way in which the sample is introduced into the instrument. Direct introduction is preferable, but it is not always possible. In some analyses of complex mixtures, the compounds must be separated prior to the MS analysis. A combination of HPLC or SFC with MS creates conditions similar to those of chemical ionization, because of the excess of other substances, such as water, alcohol or $\mathrm{CO}_{2}$. Water and alcohol are introduced into a MS instrument from the HPLC mobile phase, or $\mathrm{CO}_{2}$ is introduced from the SFC mobile phase. An excess of mobile phase from chromatographic devices quenches ion production and can also create adduct ions. GC-MS spectra give more information about the structure of a molecule than do HPLC-MS or
SFC-MS spectra. To ensure that an ion of a given mass produces a discernible peak in the spectrum, the best combination to use is GC-MS with SMB, because of short analysis times, low temperatures in the injector, transference line, and detection device, and, especially, "cold EI" and a "fly-through ion source" [12,13].

This journal welcomes discussions of all aspects of MS and Chromatography instrumentation, theory, practices, and applications, in different fields of science and industry as well as advice for improving the journal itself.

\section{REFERENCES}

[1] I. Brondz, "Historical Overview of Chromatography and Related Techniques in Analysis of Antimalarial Drug Primaquine,” Nova Science Publishers, Inc., New York, 2011, pp. 183-187.

[2] I. Brondz, K. Høiland and J. Lefler, "Supercritical Fluid Chromatography of Secondary Metabolites and MultiAnalysis by Mass Spectrometry-Ultraviolet and Corona Charged Aerosol Detection,” 12th Norwegian MS-Winter Meeting, Hafjell, 21-24 January 2007, p. 63.

[3] I. Brondz and K. Høiland, "Chemotaxonomic Differentiation between Cortinarius infractus and Cortinarius subtortus by Supercritical Fluid Chromatography Connected to a Multi-Detection System," Trends in Chromatography, Vol. 4, 2008, pp. 79-87.

[4] A. J. Dempster, "A New Method of Positive Ray Analysis,” Physical Review, Vol. 11, No. 4, 1918, pp. 316-325. doi:10.1103/PhysRev.11.316

[5] J. Mattauch and R. F. K. Herzog, "Mass Spectrograph," Zeitschrift für Physik, Vol. 89, 1934, pp. 786-795. doi:10.1007/BF01341392

[6] E. G. Johnson and A. O. Nier, “Angular Aberrations in Sector-Shaped Electromagnetic Lenses for Focusing Beams of Charged Particles,” Physical Review, Vol. 91, No. 1, 1953, pp. 10-17. doi:10.1103/PhysRev.91.10

[7] J. De Laeter and M. D. Kurz, "Alfred Nier and the Sector Field Mass Spectrometer,” Journal of Mass Spectrometry, Vol. 41, No. 7, 2006, pp. 847-854. doi:10.1002/jms.1057

[8] IUPAC, "IUPAC Compendium of Chemical Terminology,” Blackwell Science Inc., Oxford, 1997.

[9] K. T. Bainbridge, "The Equivalence of Mass and Energy," Physical Review, Vol. 44, No. 2, 1933, p. 123. doi:10.1103/PhysRev.44.123.2

[10] G. Audi, "The History of Nuclidicmasses and of Their Evaluation,” International Journal of Mass Spectrometry, Vol. 251, No. 2-3, 2006, pp. 85-94.

[11] I. Brondz, U. Klein, D. Ekeberg, D. Mantzilas, E. Hvattum, H. Schultz and F. S. Mikhailitsyn, "Nature of the Main Contaminant in the Drug Primaquine Diphosphate: GC-MS Analysis,” Asian Journal of Chemistry, Vol. 17 No. 3, 2005, pp. 1678-1688.

[12] I. Brondz, A. B. Fialkov and A. Amirav, "Analysis of Quinocide in Unprocessed Primaquine Diphosphate and Primaquine Diphosphate Tablets Using Gas Chromatography-Mass Spectrometry with Supersonic Molecular 
Beams,” Journal of Chromatography A, Vol. 1216, 2009 pp. 824-829. doi:10.1016/j.chroma.2008.11.043

[13] A. Amirav, "What Can Be Improved in GC-MS: When Multi Benefits Are Transformed into a GC-MS Revolu- tion,” International Journal of Analytical Mass Spectrometry and Chromatography (IJAMSC), Vol. 1, No. 1, 2013, pp. 31-47. 